\title{
Tsukushi expression is dependent on Notch signaling and oscillated in the presomitic mesoderm during chick somitogenesis
}

Uzzal Kumar Acharjee ${ }^{\mathrm{a}, \mathrm{b}}$, Ryu Gejima ${ }^{\mathrm{a}}$, Felemban Athary Abdulhaleem Ma, M. Asrafuzzaman Riyadh ${ }^{\mathrm{a}}$, Hideaki Tanaka ${ }^{\mathrm{a}}$, Kunimasa Ohta ${ }^{\mathrm{a}, \mathrm{c}^{*}}$

a) Division of Developmental Neurobiology, Faculty of Life Sciences, Kumamoto University, 1-1-1 Honjo, Kumamoto 860-8556, Japan

b) Program for Leading Graduate Schools HIGO (Health Life Science: Interdisciplinary and Glocal Oriented), Kumamoto University, 1-1-1 Honjo, Kumamoto 860-8556, Japan

c) Japan Agency for Medical Research and Development (AMED), Tokyo 100-0004, Japan

\footnotetext{
* Correspondence should be addressed to: Kunimasa Ohta
}

Phone: (81)-96-373-5293

Fax: +81963735293

E-mail: ohta9203@gpo.kumamoto-u.ac.jp 


\begin{abstract}
During somitogenesis, segmentation of the body axis occurs by epithelial somites budding off from the rostral end of the unsegmented presomitic mesoderm (PSM), and its molecular regulation is achieved by a molecular oscillator and signaling molecules. Tsukushi (TSK) is a unique secreted protein and involved in diverse biological cascades in vertebrate embryos by modulating several signaling pathways at the extracellular region. However, the involvement of TSK in somitogenesis remains unknown. In this study, we investigated the detailed expression patterns of TSK at different developmental stages of a chick embryo. Chick-TSK (C-TSK) is expressed in the PSM and shows an oscillation pattern with three phases. The oscillation pattern of C-TSK in the PSM is similar to that of c-Notch1 and c-hairy1, but not to c-Delta1. Our in vitro data showed that Notch signaling is necessary for the normal expression of C-TSK and that expression of C-TSK is an intrinsic property of the anterior PSM. These data suggest that TSK plays a role in chick somitogenesis.
\end{abstract}

Key words: Tsukushi, Notch signaling, Somitogenesis, Chick, Oscillation 


\section{Introduction}

Somitogenesis is one of the most fascinating and fundamental processes of vertebrate early development by which the blueprint of the vertebral column, the "somites," form from both sides of the developing neural tube [1]. These somites are transient bilateral repeated segments of the paraxial mesoderm that differentiate into the axial skeleton, body skeletal musculature, and dermis. Segmentation of the vertebrate body axis is initiated through somitogenesis, whereby epithelial somites bud off in pairs periodically from the rostral end of the unsegmented presomitic mesoderm (PSM) [2].

Somite formation involves extensive cellular readjustments, namely, cell packing and polarization, when preparing for the required mesenchymal-to-epithelial transition [3]. It is widely accepted that this process is controlled by a molecular oscillator that drives periodic waves of gene expression caudo-rostrally through the PSM with the same periodicity as somite formation [4]. Several clock genes belonging to the Wnt, Notch, shh, or FGF pathways play roles in the process of somite formation and oscillation [5]. This periodic mechanism is repeated a specific number of times until the embryo acquires a defined species-specific final number of somites at the end of the process of axis elongation [4].

Previously, we reported that Tsukushi (TSK) is a member of the Small LeucineRich Proteoglycan (SLRP) family belonging to the subclass IV [6]. TSK binds nodal/VG1 [7], BMP4/Chordin [6], FGF8 [8], Frizzled4 [9], and Delta [10] and modulates their downstream intracellular signaling pathways, indicating multiple regulatory functions of TSK during early development. TSK is also involved in anterior commissure formation in the mouse brain [11]. TSK, therefore, play multiple 
roles as a signaling mediator at the extracellular region during many key biological events [12].

In this study, we investigated the detailed expression pattern of C-TSK at different developmental stages of chick somitogenesis. Using an in situ hybridization technique, we found that C-TSK is expressed in the PSM and shows an oscillation similar to that of c-hairy1 and c-Notch1, but not to c-Delta1. Further, an in vitro culture assay showed that Notch signaling is necessary for the normal expression of C-TSK and that the expression of C-TSK is an intrinsic property of the PSM. Our results suggest that TSK plays a role in chick somitogenesis with oscillation by interacting Notch signaling.

\section{Materials and methods}

\subsection{Chick}

Chick eggs were incubated at $38^{\circ} \mathrm{C}$ in a humidified incubator. The embryos were staged according to the developmental table of Hamburger and Hamilton [13]. The stage specific (stage 7 , stage 8 , stage 10 , stage 15 , stage 18 , stage 23 ) embryos were used for in situ hybridization. The newly formed somites were designated S1, S2, S3, the forming somite was designated S0, and the one somite length unsegmented PSM was designated S-1. All experiments on chick were conducted in accordance with the guidelines of the Center for Animal Resources and Development (CARD), Kumamoto University, Japan.

\subsection{In situ hybridization}

In situ hybridization was performed as previously described [6] to assess C-TSK, c-hairy1, c-Notch1 and c-Delta1 expression patterns in the presomitic mesoderm. For 
experiment on oscillation pattern, stage 15 (somites 25) embryos were selected by counting the somite numbers, cut the embryos into two equal halves, and complementary pairs were used for in situ hybridization. Sense and antisense riboprobes labeled with Digoxigenin (DIG) were synthesized from the corresponding DNA constructs [14]. A color reaction was initiated with $337.5 \mu \mathrm{g} / \mathrm{ml}$ 4-Nitro blue tetrazolium (NBT, Roche) and 5-Bromo-4-chloro-3-indolyl-phosphate (BCIP, Roche) in freshly prepared Alkaline Phosphatase (AP) buffer (0.1 M Tris-HCL, pH 9.5, 0.1M $\mathrm{NaCl}, 50 \mathrm{mM} \mathrm{MgCl} 2,1 \%$ Tween-20, and $0.48 \mathrm{mg} / \mathrm{ml}$ levamisole hydrochloride).

\subsection{Chick embryo and explants in vitro culture}

Chick embryos ranging from 15 to 20 somites were cultured in L15 medium (L15 Leibovitz medium, Gibco) supplemented with 15\% fetal calf serum (FCS) and $0.1 \%$ gentamycin (Sigma Aldrich) in a roller incubator at $37^{\circ} \mathrm{C}$. For pharmacological inhibition of signaling pathways, embryos were cultured for 9 hours in $10-100 \mu \mathrm{M} \mathrm{N}$ [N-(3,5-difluorophenacetyl)-L-alanyl]-S-phenylglycine t-butyl ester, (DAPT, Calbiochem). The DAPT was diluted in culture medium from stock solutions in dimethylsulphoxide (DMSO) to maintain a final concentration of $1 \%$. For in vitro culture of the explants (stage 14) containing PSM, embryos were isolated from surrounding tissues and the cultured (0-6 hours) embryos were hybridized with C-TSK probes.

\subsection{Image Analysis}

Embryos processed for whole mount in situ hybridization were photographed, using an Olympus DP70 digital camera coupled with a Leica MZFLIII stereomicroscope equipped with a DP Controller image manager (Olympus 
Corporation, Japan). Cryosectioned samples were photographed using a Keyence BZ9000 fluorescence microscope (Keyence Corporation, Japan).

\section{Results}

\subsection{C-TSK is expressed in PSM during chick somitogenesis}

During chick embryogenesis, C-TSK is expressed in various tissues, including the limb bud, nasal pit, and PSM [7,8]. To determine TSK mRNA expression in detail in the PSM and newly forming somites, we examined the expression pattern of C-TSK in the PSM of different developmental stages of chick embryos (Fig. 1). C-TSK was

detected beginning at stage 7 , where the first somite pair originated from both sides of the neural tube (Fig. 1A). As development proceeded, the number of somites increased and the most mature somites reached near the neck of the embryo, while the newly formed somites were located near the posterior part of the embryo (Fig. 1B and C). At stage 15, the expression of C-TSK was detected in the anterior PSM (Fig. 1D). During stage 18, C-TSK is also expressed in the newly forming somites and the PSM that reached beyond the leg bud (Fig. 1E). At stage 23, C-TSK was expressed in the somites and PSM near the tip of the tail and also in the wing/leg buds (Fig. 1F). In a sagittal section of the last formed somite and the PSM at stage 15, C-TSK was expressed as two bands, a narrow band in the caudal half of the newly formed somite ' $\mathrm{S} 1$ ', a thick band in the caudal half of the forming somite ' $\mathrm{S} 0$ ' and in the unsegmented posterior PSM (Fig. 1G). These results suggest that, during embryonic development, C-TSK is expressed at different stages in the chick PSM.

\subsection{The expression of C-TSK oscillates in the chick anterior PSM}


To determine whether C-TSK expression oscillates, we compared its expression with c-hairy1, c-Notch1, and c-Delta1 mRNA in the chick anterior PSM (Fig. 2). The expression of c-hairy1 mRNA in the PSM oscillates with the same periodicity as the somite forms and one complete cycle of c-hairy1 oscillation represents the formation of one somite [15]. Importantly, c-hairy1 is a target of the Notch signaling cascade [15]. In our study, the expression patterns were divided into three phases (phases IIII) as per somite formation periodicity in a one cycle and a new somite is formed from the rostral-most part of the PSM. At phase I, c-hairy1 was visible as two bands, one narrow band in the caudal part of the forming somite S0, and another thick band that extended caudally from the S-1 to the tail area (Fig. 2A). In contrast, C-TSK expression started from the caudal part of the newly forming somite S0 as a single and extended band along the PSM (Fig. 2A). At phase II, with the progression of somite formation, C-TSK expression was detected as two bands: the very narrow band was detected in the caudal half of the forming somite S0, while the thick band was detected in the caudal part of the S-1. In the complementary band, c-hairy1 expanded anteriorly and the caudal-most part vanished (Fig. 2B). At phase III, this wave front like expression of both c-hairy1 and C-TSK eventually narrowed and reduced towards the S0 (Fig. 2C). In this phase, the formation of somite S0 was nearly completed.

Next, we compared C-TSK and c-Notch1 expression in the anterior PSM (Fig. 2DF). At phase I, c-Notch1 was expressed weakly at the caudal region of newly formed somite S1, in the PSM it expresses weakly from the forming somite S0, and extended strongly from the unsegmented PSM S-1 (Fig. 2D). At phase II, c-Notch1 was expressed at the caudal half of S1 and extended to S0, S-1, and posterior PSM (Fig. 2E). At phase III, c-Notch1 expression moved more anterior and posterior part of the PSM. Here, c-Notch1 occupied the entire last formed somite S1, then it expressed 
posteriorly beyond the S-1 (Fig. 2F). In our observations, we found upregulated expression of c-Notch1 in the anterior part of the PSM in compare to posterior part. As development proceeded, the expression pattern of c-Notch1 was gradually downregulated towards the posterior part of the PSM. In contrast, C-TSK expression was observed as a distinct band along the A-P axis. S0 and S-1 at phase II became S1 and S0, respectively, at phase III. C-TSK expression is not visible at the last formed somite $\mathrm{S} 1$ at phase III (Fig. 2C, F, I). In our experiment the expression pattern of chairy1 and c-Notch1 in chick somitogenesis has similarity with the previous study [16].

Finally, we compared C-TSK and c-Delta1 in the anterior PSM (Fig. 2G-I). Expressions of both C-TSK and c-Delta1 were robust in the anterior PSM during somitogenesis. We found that c-Delta1 was expressed in a very distinct domain in chick PSM, which is similar with the previous study [16]. But, c-Delta1 expression was stable at all phases in the anterior PSM, and did not show the dynamic oscillation pattern of C-TSK, c-hairy1, and c-Notch1. This comparative expression pattern suggests that C-TSK exhibits a dynamic oscillation in the PSM.

3.3. Notch signaling is necessary for the normal expression of C-TSK and the expression of C-TSK is an intrinsic property of the PSM

To examine how Notch signaling interacts with C-TSK in chick somite development, we used a pharmacological inhibition approach by the Notch inhibitor DAPT (Fig. 3A-D). We isolated unsegmented mesoderm at embryonic stage 14, cultured in the presence or absence of DAPT, and analyzed target genes either with DAPT (Fig. 3B and D) or without (Fig. 3A and C). To confirm that DAPT-mediated inhibition was not due to a usual disruption of the PSM identity patterning, we 
checked the expression of the PSM marker T-box transcription factor 6 (Tbx6). After 9 hours of cultivation, C-TSK (Fig. 3A and B) and Tbx6 (Fig. 3C and D) expression patterns were analyzed. C-TSK expression at the PSM in medium containing DAPT was reduced in comparison to the control (DMSO).

To determine whether the expression of C-TSK is intrinsically controlled by the PSM, we performed an explant culture assay and an in situ hybridization experiment (Fig. 3E-G). In our study, 14-20 somites stage chick PSM was detached from the surrounding tissues, cultured for 0 (Fig. 3E), 3 (Fig. 3F), or 6 (Fig. 3G) hours, and then hybridized with a C-TSK probe. In this experiment, the ectoderm and endoderm were not associated with the PSM. Under these conditions, we did not see any segments, but the expression of C-TSK was sustained. The expression of C-TSK was progressively downregulated and shifted to the more caudal parts of the explants (Fig. 3G). These results suggest that Notch signaling is necessary for the normal expression of C-TSK in the chick PSM and expression of C-TSK is autonomously regulated by the unsegmented mesoderm or PSM. Therefore, the expression of C-TSK is an intrinsic property in PSM development.

\section{Discussion}

Early patterning of the body during animal development is a fundamental process to subsequent events including cell differentiation, tissue and organ formation, and correct function of the adult body [17]. To trigger this rhythmic production of somitic segments, three major signaling pathways, Notch, Wnt, and FGF integrate into a molecular network that generates a traveling wave of gene expression along the embryonic axis, called the "segmentation clock" [18]. In addition to these signaling pathways, TSK, a member of the extracellular proteoglycan gene family [19] also 
plays a role in somitogenesis. As TSK is expressed in the PSM and is involved in the above signaling pathways [12], it is plausible to expect the involvement of TSK in somitogenesis. In this study, the in situ hybridization data provide evidences for the involvement of C-TSK in different stages of somitogenesis. This detailed expression pattern of chick embryogenesis denotes the timing, location, and pattern of C-TSK expression that is required for somite formation and progression.

Figure 4 compared and summarized the expression pattern of C-TSK and Notch signaling molecules during somitogenesis. During early phase, c-hairy1 was first expressed in the posterior PSM, and then gradually, it is expressed in the more anterior domains. C-TSK also showed caudal-to-rostral shifting of expression and refined in later phases to retain the wave-like expression. Though the c-hairy1 and CTSK expression domains and band size is not similar, both of them showed a dynamic expression pattern in the anterior PSM. The involvement of the Notch signaling pathway in somitogenesis was first revealed by the finding that Notch1 and its ligand Delta-like 1 (D111), which are highly expressed in the PSM of mouse embryos, play important roles in inter- and intra-somitic boundary formation [20-22]. In our observation, c-Notch1 expression was upregulated in anterior domains of the PSM. This oscillation of c-Notch1 is consistent with the pattern of C-TSK expression during early development. Both C-TSK and c-Delta1 expression was seen robustly in the anterior PSM during somitogenesis. Though c-Delta1 was expressed in a very distinct domain in chick PSM, expression was stable in the anterior PSM, and did not show a pattern of oscillation similar to C-TSK, c-hairy1, and c-Notch1. Therefore, C-TSK expression seems unlikely to be related to c-Delta1 expression and this comparison supports the oscillatory behavior of C-TSK in the anterior PSM. 
Our in vitro culture experiment and pharmacological inhibition data showed that Notch signaling is essential for the normal expression of C-TSK. The regulation of clock gene oscillations by the Notch signaling pathway plays a key role in the molecular mechanism of the segmentation clock in a number of different vertebrate species [23]. C-TSK expression was downregulated in the presence of DAPT, which pharmacologically inhibited the Notch signaling pathway. The PSM still possesses mesenchymal congruity by expressing the PSM marker Tbx6 that has previously been shown to be a Wnt target in the mouse PSM [24]. Inhibition of Notch may cause residual Wnt activity to upregulate Tbx6 expression. As Notch is an integral part of the oscillator and segmentation critically depends on its signaling, C-TSK expression also relies on Notch signaling in the anterior PSM. Therefore, C-TSK expression was reduced in embryos incubated in DAPT to inhibit the corresponding pathways. It is well known that somitogenesis can proceed in vitro, provided that the ectoderm and endoderm remain associated with the PSM [25]. Moreover, in complete isolation, the PSM does not form any somites but the cyclic expression of c-hairy1 is maintained [26]. In our study, no somites or segments were formed, but that the expression of CTSK was sustained. The expression of C-TSK was autonomously regulated by the unsegmented mesoderm and the expression of C-TSK intrinsic to the PSM. However, it is not yet clear whether the periodic gene expression of C-TSK is associated with the segmentation clock due to the cyclic gene network. TSK directly binds several signaling molecules in the extracellular space through protein-protein interactions, and it functions as an extracellular modulator of signaling cascades by enhancing or inhibiting their activity during early embryonic development [12]. Considering this, we hypothesize that TSK also maintains its own gene network and acts as an 
extracellular signaling mediator for somite formation and oscillations in gene expression. 


\section{Acknowledgements}

We thank to Yoshiko Takahashi, Yuichi Oike, and Naofumi Ito for their critical comments; Mitsue Kumamaru, Kazumi Yamada, and Ai Hamashima for technical assistance; and all members of our labs for their valuable help. This work was supported by the Precursory Research for Embryonic Science and Technology program of the Japan Science and Technology Agency (K.O.), and Grants-in-Aid for Scientific Research on Molecular Brain Science from the Ministry of Education Culture, Sports, Science, and Technology of Japan (K.O.), the Global COE Program (Cell Fate Regulation Research and Education Unit) (H.T.), and the Program for Leading Graduate Schools "HIGO (Health life science: Interdisciplinary and Glocal Oriented)", MEXT, Japan (U.K.A.). 


\section{References}

[1] Y. Takahashi, Somitogenesis in Vertebrate Development, eLS. 2005. DOI: 10.1038/npg.els.0003820.

[2] Z. Ferjentsik, S. Hayashi, J.K. Dale, Y. Bessho, A. Herreman, B. De Strooper, et al., Notch is a critical component of the mouse somitogenesis oscillator and is essential for the formation of the somites, PLoS Genet. 5 (2009) e1000662.

[3] T.P. Resende, R.P. Andrade, I. Palmeirim, Timing embryo segmentation: dynamics and regulatory mechanisms of the vertebrate segmentation clock, Biomed Res Int. 2014 (2014) 718683-12.

[4] G. Tenin, D. Wright, Z. Ferjentsik, R. Bone, M.J. McGrew, M. Maroto, The chick somitogenesis oscillator is arrested before all paraxial mesoderm is segmented into somites, BMC Dev. Biol. 10 (2010) 24.

[5] S. Gibb, A. Zagorska, K. Melton, G. Tenin, I. Vacca, P. Trainor, et al., Interfering with Wnt signalling alters the periodicity of the segmentation clock, Dev. Biol. 330 (2009) 21-31.

[6] K. Ohta, G. Lupo, S. Kuriyama, R. Keynes, C.E. Holt, W.A. Harris, et al., Tsukushi functions as an organizer inducer by inhibition of BMP activity in cooperation with chordin, Dev. Cell. 7 (2004) 347-358.

[7] K. Ohta, S. Kuriyama, T. Okafuji, R. Gejima, S.-I. Ohnuma, H. Tanaka, Tsukushi cooperates with VG1 to induce primitive streak and Hensen's node formation in the chick embryo, Development. 133 (2006) 3777-3786.

[8] S.A. Morris, A.D. Almeida, H. Tanaka, K. Ohta, S.-I. Ohnuma, Tsukushi modulates Xnr2, FGF and BMP signaling: regulation of Xenopus germ layer formation, PLoS ONE. 2 (2007) e1004.

[9] K. Ohta, A. Ito, S. Kuriyama, G. Lupo, M. Kosaka, S.-I. Ohnuma, et al., 
Tsukushi functions as a Wnt signaling inhibitor by competing with Wnt2b for binding to transmembrane protein Frizzled4, Proc. Natl. Acad. Sci. U.S.A. 108 (2011) 14962-14967.

[10] S. Kuriyama, G. Lupo, K. Ohta, S.-I. Ohnuma, W.A. Harris, H. Tanaka, Tsukushi controls ectodermal patterning and neural crest specification in Xenopus by direct regulation of BMP4 and X-delta-1 activity, Development. 133 (2006) 75-88.

[11] A. Ito, Y. Shinmyo, T. Abe, N. Oshima, H. Tanaka, K. Ohta, Tsukushi is required for anterior commissure formation in mouse brain, Biochem. Biophys. Res. Commun. 402 (2010) 813-818.

[12] K. Ohta, The Role of Tsukushi as an Extracellular Signaling Coordinator, New Principles in Developmental Processes. (2014) 227-238.

[13] V. HAMBURGER, H.L. HAMILTON, A series of normal stages in the development of the chick embryo, J. Morphol. 88 (1951) 49-92.

[14] M.A. Riyadh, Y. Shinmyo, K. Ohta, H. Tanaka, Inhibitory effects of draxin on axonal outgrowth and migration of precerebellar neurons, Biochem. Biophys. Res. Commun. 449 (2014) 169-174.

[15] A. Aulehla, B.G. Herrmann, Segmentation in vertebrates: clock and gradient finally joined, Genes Dev. 18 (2004) 2060-2067.

[16] I. Palmeirim, J. Dubrulle, D. Henrique, D. Ish-Horowicz, O. Pourquié, Uncoupling segmentation and somitogenesis in the chick presomitic mesoderm, Dev. Genet. 23 (1998) 77-85.

[17] Y. Takahashi, N. Osumi, N.H. Patel, Body patterning, Proc. Natl. Acad. Sci. U.S.A. 98 (2001) 12338-12339.

[18] O. Pourquié, Vertebrate segmentation: from cyclic gene networks to scoliosis, 
Cell. 145 (2011) 650-663.

[19] R.V. Iozzo, L. Schaefer, Proteoglycan form and function: A comprehensive nomenclature of proteoglycans, Matrix Biol. 42 (2015) 11-55.

[20] R.A. Conlon, A.G. Reaume, J. Rossant, Notch1 is required for the coordinate segmentation of somites, Development. 121 (1995) 1533-1545.

[21] M. Hrabě de Angelis, J. McIntyre, A. Gossler, Maintenance of somite borders in mice requires the Delta homologue DII1, Nature. 386 (1997) 717-721.

[22] Y. Sato, K. Yasuda, Y. Takahashi, Morphological boundary forms by a novel inductive event mediated by Lunatic fringe and Notch during somitic segmentation, Development. 129 (2002) 3633-3644.

[23] P.C.G. Rida, N. Le Minh, Y.J. Jiang, A Notch feeling of somite segmentation and beyond, Dev. Biol. 265 (2004) 2-22.

[24] W.C. Dunty, K.K. Biris, R.B. Chalamalasetty, M.M. Taketo, M. Lewandoski, T.P. Yamaguchi, Wnt3a/beta-catenin signaling controls posterior body development by coordinating mesoderm formation and segmentation, Development. 135 (2008) 85-94.

[25] D.S. Packard, The influence of axial structures on chick somite formation, Dev. Biol. 53 (1976) 36-48.

[26] I. Palmeirim, D. Henrique, D. Ish-Horowicz, O. Pourquié, Avian hairy gene expression identifies a molecular clock linked to vertebrate segmentation and somitogenesis, Cell. 91 (1997) 639-648. 


\section{Figure legends}

Fig. 1. Expression of C-TSK in the PSM at different stages of chick embryos. (A-F) Whole mount in situ hybridization. C-TSK is detected at stage 7 (A), stage 8 (B), stage $10(\mathrm{C})$, stage $15(\mathrm{D})$, stage $18(\mathrm{E})$, and stage 23 (F) (violet color in all stages). The black arrowheads show the PSM expressing C-TSK. The boxed areas are magnified views of the PSM. (G) Sagittal section of the PSM at stage 15, where CTSK is expressed weakly in the caudal half of the newly formed somite ' $\mathrm{S} 1$ ', strongly from the caudal half of the forming somite ' $\mathrm{S} 0$ ' and in the unsegmented posterior PSM. ps, primitive streak; tb, tail bud; wb, wing bud; lg, leg bud. Scale bars: $150 \mu \mathrm{m}$ in $\mathrm{A} ; 300 \mu \mathrm{m}$ in $\mathrm{B} ; 400 \mu \mathrm{m}$ in $\mathrm{C}-\mathrm{E} ; 2 \mathrm{~mm}$ in $\mathrm{F} ; 50 \mu \mathrm{m}$ in $\mathrm{G}$.

Fig. 2. Comparison of C-TSK with c-hairy1 (A-C), c-Notch1 (D-F), and c-Delta1 (GI) in the chick anterior PSM at stage 15. (A) At phase I, C-TSK is expressed as a single band and c-hairy1 expressed as two bands in S0, S-1. (B) At phase II, C-TSK and c-hairy1 expressed as two bands at S0 and S-1 (C) At phase III, expression of both C-TSK and c-hairy1 are narrowed. (D) At phase I, C-TSK express along the S-1, while c-Notch1 is weakly expressed at the caudal half of S1, S0, but express strongly from the of S-1. (E) At phase II, C-TSK express as two bands, S0 and S-1, c-Notch1 is expressed in the caudal half of S1, and entire S0, S-1 (F) At phase III, C-TSK narrowed but c-Notch1 expression covers the S1, S0, S-1. (G-I) C-TSK oscillates at S0 and S-1, while c-Delta1 expression does not show oscillations at the anterior PSM. Scale bars: $150 \mu \mathrm{m}$ in A-C; $100 \mu \mathrm{m}$ in D-F; $100 \mu \mathrm{m}$ in G-I.

Fig. 3. C-TSK expression is dependent on Notch signaling and intrinsically regulated by the unsegmented mesoderm. Embryos at stage 14 were cultured at $37^{\circ} \mathrm{C}$ with 
DAPT $(B, n=11 ; D, n=9)$ or control $(A, n=10 ; C, n=13)$. After 9 hours of culture, C-TSK (A, B) and Tbx6 (C, D) expression patterns were analyzed by in situ hybridization. C-TSK expression in medium containing DAPT (B) was diminished in comparison with the control (A). (E-G) PSM at the 14-20 somites stage was isolated from the surrounding tissues and was cultured for 0 (A), 3 (B), or 6 (C) hours. Note that the expression of C-TSK was maintained and progressively shifted to the more caudal parts of the explants. $r$, rostral; c, caudal. Scale bar: $300 \mu \mathrm{m}$ in A-D; $200 \mu \mathrm{m}$ in E-G.

Fig. 4. Schematic comparison of the oscillatory expression patterns in the anterior PSM. At phases I to III, C-TSK (red) expression is observed as dynamic oscillation. At phases I to II, c-hairy1 (violet) was expressed as two extended bands, but at phase III as a single narrow band. At phase I, c-Notch1 (green) is weakly expressed at S0, but dispersed from the caudal half of the anterior PSM. Later, it extends anteriorly up to S1. At phase III, c-Notch1 is extended beyond the S-1. In all three phases, c-Delta1 expression (blue) is characterized as stable and does not show oscillations at the anterior PSM. In phase III, somite S0 is nearly ready to bud off as a new somite S1. The end of this phase starts again as phase I. 
Figure -1

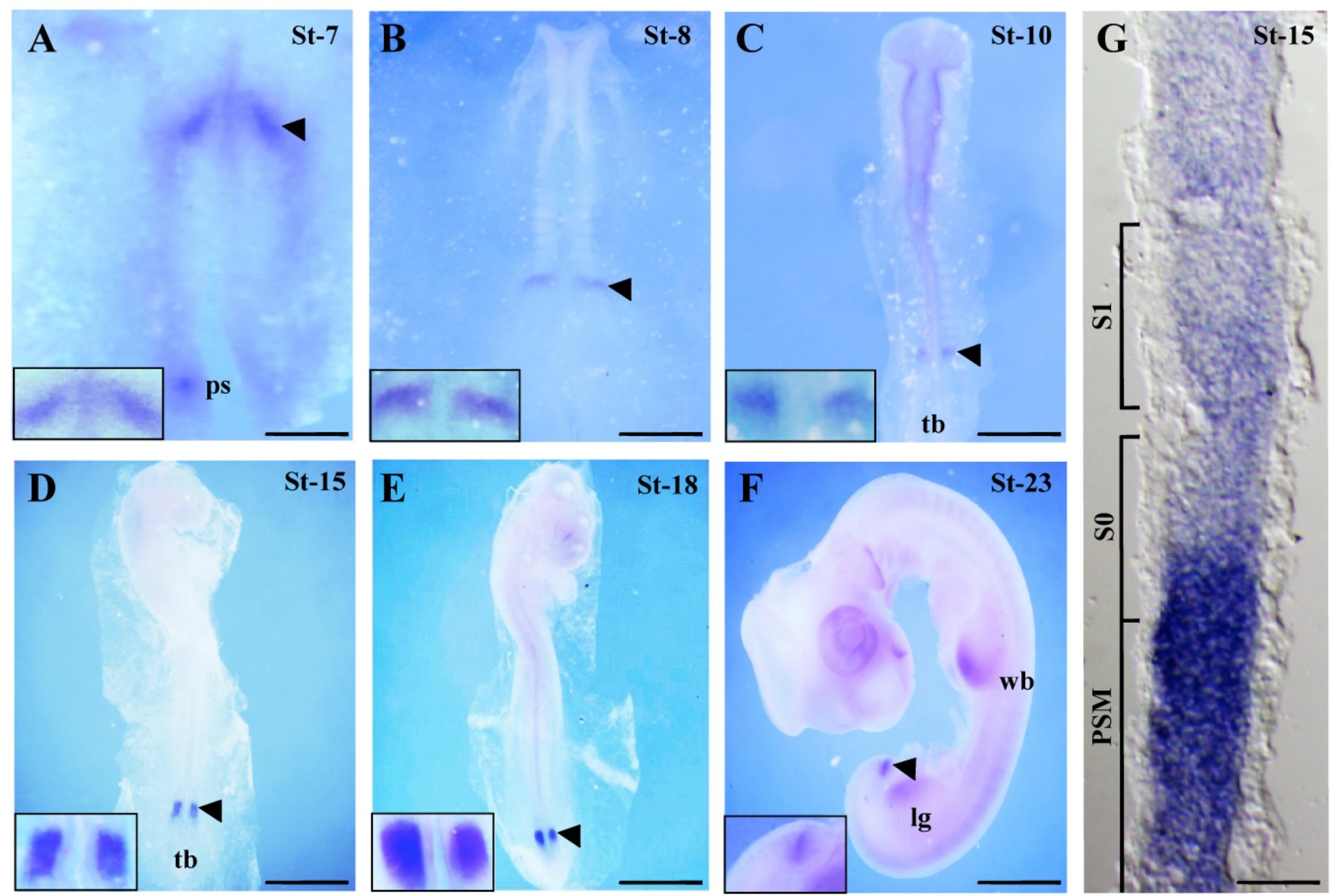


Figure -3

\section{DMSO}

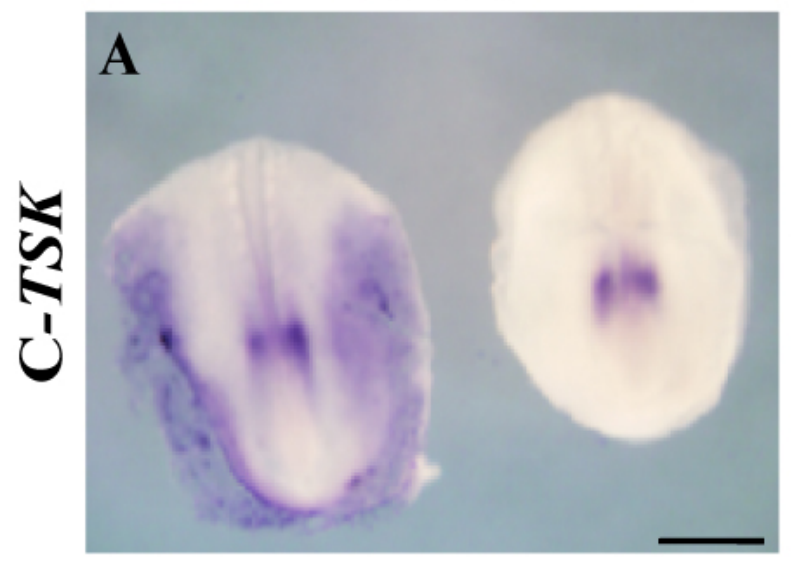

$\frac{0}{0}$

C
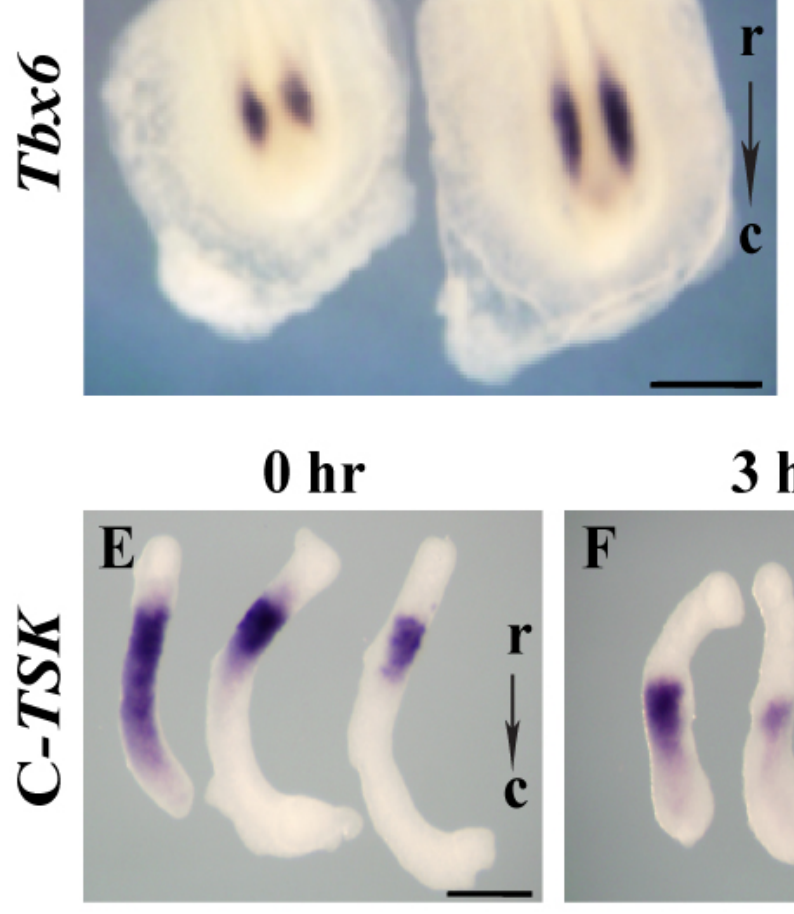

DAPT

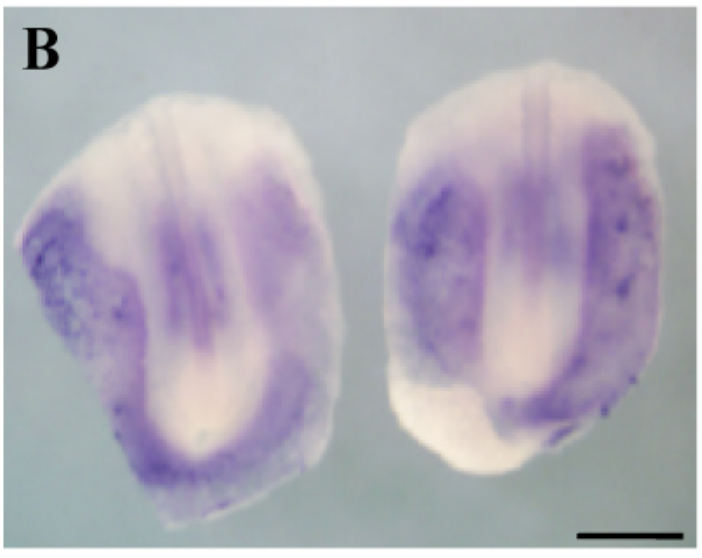

D

3 hrs

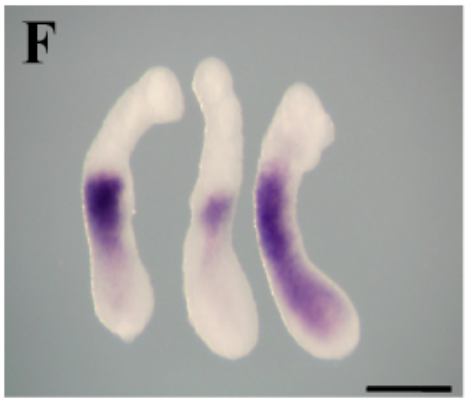

6 hrs

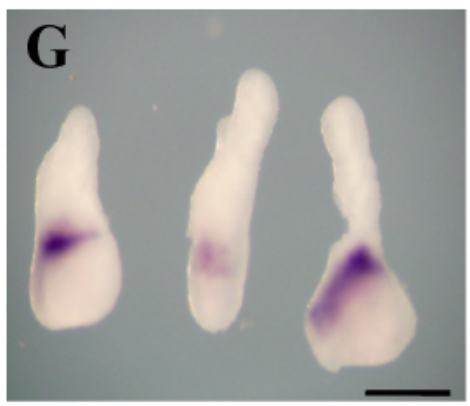




\section{Figure -4}

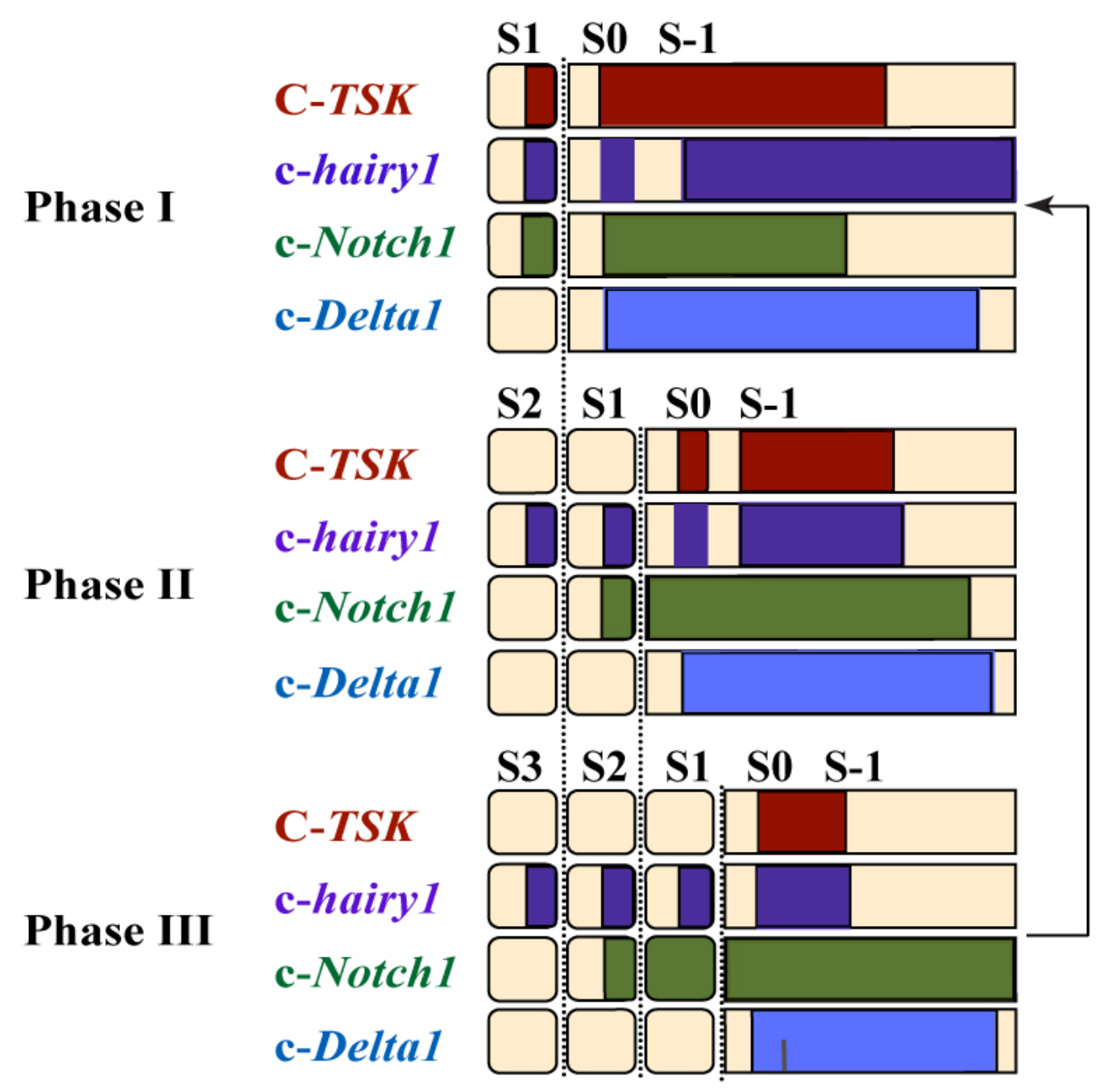

Anterior $\longrightarrow$ Posterior 